\title{
Entangled two-photon absorption
}

\section{spectroscopy}

\author{
Frank Schlawin, ${ }^{*} \dagger$ Konstantin E. Dorfman, ${ }^{\ddagger}$ and Shaul Mukamel \\ $\dagger$ Department of Physics, University of Oxford, Oxford OX1 1PU, United Kingdom \\ $\ddagger$ State Key Laboratory of Precision Spectroscopy, East China Normal University, Shanghai \\ 200062, China \\ \Chemistry Department and Physics and Astronomy Department, University of California, \\ Irvine, USA \\ E-mail: frank.schlawin@physics.ox.ac.uk
}

Conspectus
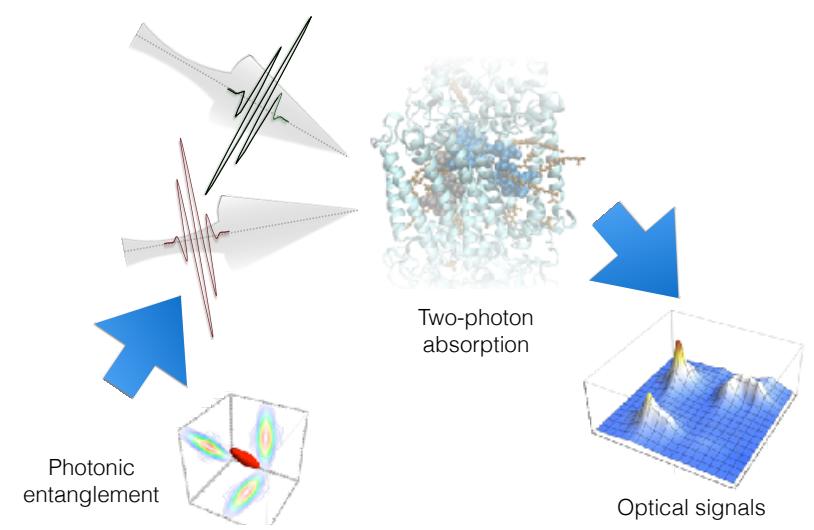

The application of quantum states of light such as entangled photons, created e.g. by parametric downconversion, has experienced tremendous progress in the almost 40 years since their first experimental realization. Initially, they were employed in the investigation of the foundations of quantum physics, such as the violation of Bell's inequalities and studies of quantum entanglement. They later emerged as basic platforms 
for quantum communication protocols, and, in the recent experiments on single-photon interactions, in photonic quantum computation. These applications aim at the controlled manipulation of the photonic degrees of freedom, and therefore rely on simple models of matter, where the analysis is simpler. Furthermore, quantum imaging with entangled light can achieve enhanced resolution, and quantum metrology can overcome the shot noise limit for classical light.

This account focuses on an entirely different emerging class of applications using quantum light as a powerful spectroscopic tool to reveal novel information about complex molecules. These applications utilize two appealing properties of quantum light its distinct intensity fluctuations, and its nonclassical bandwidth properties. As will be discussed in this account, these give rise to new and surprising behavior of nonlinear optical signals. Nonclassical intensity fluctuations can enhance nonlinear optical signals relative to linear absorption. For instance, the two-photon absorption of entangled photon pairs scales linearly (rather than quadratically) in the photon flux just like a single photon absorption. This enables nonlinear quantum spectroscopy of photosensitive, e.g. biological, samples at low light intensities. We will discuss how the two-photon absorption cross section becomes a function of the photonic quantum state, which can be manipulated by properties of the entangled photon pairs. In addition, the quantum correlations in entangled photon states further influence the nonlinear signals in a variety of ways. Apart from affecting the signal's scaling with intensity, they also constitute an entirely new approach to shaping and controlling excitation pathways in molecular aggregates in a way that cannot be achieved with shaped classical pulses. This is because in between the two absorption events in entangled two-photon absorption, the light and material system are entangled. Classical constraints for the simultaneous time and frequency resolution can thus be circumvented, since the two are not Fourier conjugates.

Here we review the simplest manifestation of quantum light spectroscopy, twophoton absorption spectroscopy with entangled photons. This will allow us to discuss exemplarily the impact of quantum properties of light on a nonlinear optical signal, 
and explore the opportunities for future applications.

\section{Introduction}

Quantum entanglement refers to a curious property of the Hilbert space geometry of manybody systems: It is associated with many-body quantum states which cannot be written as the products of the constituents' wavefunctions, and consequently the particles are strongly correlated in a distinct quantum-mechanical way as we describe below. The concept of entanglement, and the non-local correlations associated with it, was first pointed out in the famous paper by Einstein, Podolsky and Rosen, ${ }^{1}$ who used it to question the foundations of quantum mechanics. Its implications for our understanding of the nonlocality of quantum mechanics are hard to underestimate. But besides, as later formalised by Bell, ${ }^{2}$ its main corollary for possible applications consists in the insight that there are quantum states featuring strong correlations in any measurement basis - as opposed to classical correlations which are basisdependent. For instance, a two-spin Bell state such as $\left|\Phi^{+}\right\rangle=\left(\left|\uparrow_{1} \uparrow_{2}\right\rangle+\left|\downarrow_{1} \downarrow_{2}\right\rangle\right) / \sqrt{2}$ shows perfect correlations between the two spins, regardless of whether one measures correlations in the up-/down-basis $\left|\uparrow_{1}\right\rangle,\left|\downarrow_{1}\right\rangle$ or in the plus-/minus-basis $\left| \pm_{1}\right\rangle=\left(\left|\uparrow_{1}\right\rangle \pm\left|\downarrow_{1}\right\rangle\right) / \sqrt{2}$ of spin no. 1: In the latter basis, the Bell state retains its structure, $\left|\Phi^{+}\right\rangle=\left(\left|+{ }_{1}+{ }_{2}\right\rangle+\left|-{ }_{1}-{ }_{2}\right\rangle\right) / \sqrt{2}$. Therefore, the measurement of spin 1 determines the state of spin 2 . If the result is $\left|\uparrow_{1}\right\rangle$, we know that spin 2 is in the state $\left|\uparrow_{2}\right\rangle$ (likewise for $\left|\downarrow_{1}\right\rangle$ ). Also, if the result is $\left|+_{1}\right\rangle$, we know that spin 2 is in the state $\left|+_{2}\right\rangle$ (and likewise for $\left|-_{1}\right\rangle$, of course). In contrast, classically correlated states $\left[\right.$ e.g. $\rho=\left(\left|\uparrow_{1} \uparrow_{2}\right\rangle\left\langle\uparrow_{1} \uparrow_{2}|+| \downarrow_{1} \downarrow_{2}\right\rangle\left\langle\downarrow_{1} \downarrow_{2}\right|\right) / 2$ which is obtained from $\left|\Phi^{+}\right\rangle\left\langle\Phi^{+}\right|$ by removing its off-diagonal matrix elements in the up-/down-basis] can only show perfect correlations in a specific basis. Measuring $\rho$ in the up-/down-basis of spin no. 1, we obtain the reduced density matrix $\rho_{\text {red }}=\left\langle\uparrow_{1}|\rho| \uparrow_{1}\right\rangle=\left|\uparrow_{2}\right\rangle\left\langle\uparrow_{2}\right|$, so this measurement determines

the state of the second spin as shown above. Yet measuring $\rho$ in the plus-/minus-basis, we obtain $\rho_{\text {red }}=\left(\left|+_{2}\right\rangle\left\langle+_{2}|+|-_{2}\right\rangle\left\langle-_{2}\right|\right) / 2$ which leaves spin no. 2 undetermined. This feature 
will be important in spectroscopy, where entangled photon states can simultaneously feature strong correlations in time and frequency domains, while classical correlations only show up in one domain.

Photons can be entangled in different degrees of freedom: their wavevectors, frequency, time, or polarizations. It was with polarization-entangled photons that the violation of Bell's inequalities was first demonstrated conclusively. ${ }^{3-5}$ These experiments paved the way for the currently most intensive field of research in the application of entangled photons - quantum cryptography. ${ }^{6}$ Further proposals aim at employing entangled photons in imaging, ${ }^{7}$ lithography, ${ }^{8}$ or phase estimation, ${ }^{9}$ where frequency correlations increase the spatial resolution. These proposals can, however, often be realized with classical correlations as well. ${ }^{10-12}$

The interest in employing entangled photons as spectroscopic tools was first raised in Refs., ${ }^{13-15}$ where the authors theoretically predicted a linear rather than quadratic scaling of the two-photon absorption (TPA) rate with the pump photon intensity. The experimental verification of this effect in atomic ${ }^{16-18}$ and molecular samples ${ }^{19-22}$ established entangled photons as interesting light sources for nonlinear spectroscopy with low photon fluxes. Subsequent theoretical proposals for entangled virtual state spectroscopy, ${ }^{23}$ or entanglementinduced two-photon transparency ${ }^{24}$ pointed out the highly unusual bandwidth properties of entangled photons, which will be discussed below. They also form the basis for pump-probe spectroscopy schemes ${ }^{25,26}$ or two-dimensional spectroscopy ${ }^{27,28}$ involving entangled photons.

Here we focus on experimentally relevant aspects of entangled photon absorption, while keeping the theoretical background to a necessary minimum. More comprehensive reviews can be found in Refs. ${ }^{29,30}$ We will discuss the interrelation between the photon flux produced by an entangled photon source, the inherent quantum correlations, and the induced TPA signal in atomic and molecular samples. This is followed by a discussion of TPA in molecular aggregates, where many excitation pathways interfere, and nonradiative relaxation processes reshuffle populations. 


\section{Multimode quantum light - generation and detection}
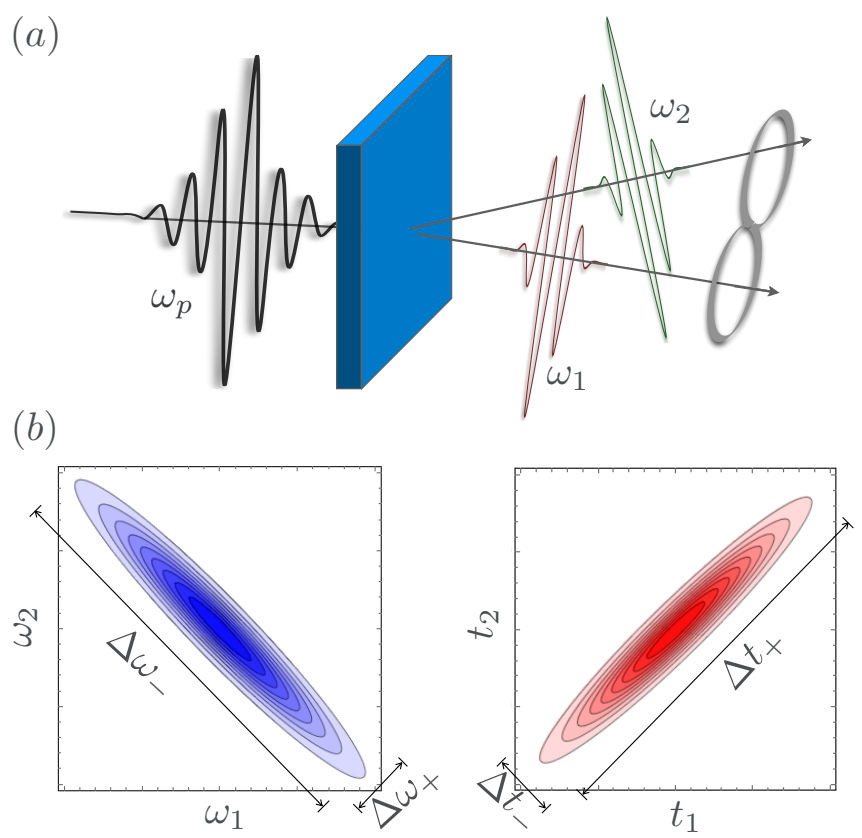

Figure 1: (a) In parametric downconversion, a photon from a strong pump beam with frequency $\omega_{p}$ (black) splits into an entangled pair of photons with frequencies $\omega_{1}$ and $\omega_{2}$. Here we depict non-collinear downconversion, but in this review we only focus on the bandwidth properties of entangled photons, and do not discuss their spatial properties. (b) Left panel: Schematic sketch of the absolute value of a typical two-photon wavefunction in frequency domain, $\Psi\left(\omega_{1}, \omega_{2}\right)$. Right panel: The absolute value of the corresponding wavefunction in time domain, $\tilde{\Psi}\left(t_{1}, t_{2}\right)$.

Before discussing the signatures of quantum properties of light in spectroscopic signals, we first briefly survey the quantum theory of the detection and characterisation of light, and the creation of entangled photon pairs.

\subsection{Parametric downconversion}

Parametric downconversion (Fig. 1(a)) is a stimulated nonlinear optical process induced by the interaction of a pump laser with a nonlinear medium. A high-frequency pump photon is split into into a pair of entangled photons - historically denoted signal and idler, respectively. Generally, one distinguishes collinear downconversion, where the entangled photons 
are created with the same propagation direction as the pump beam, and non-collinear downconversion (as sketched in Fig. 1(a)), where they are generated in cones around the pump beam. Even though by now entangled photons have been created in a broad variety of systems, the creation of time-frequency entanglement always relies on the interplay between the conservation of energy and momentum. Denoting the frequency of the pump photon $\omega_{p}$, and those of signal and idler photons $\omega_{1}$ and $\omega_{2}$, respectively, the process must conserve energy,

$$
\omega_{p}=\omega_{1}+\omega_{2}
$$

and momentum

$$
\mathbf{k}_{p}\left(\omega_{p}\right)=\mathbf{k}_{1}\left(\omega_{1}\right)+\mathbf{k}_{2}\left(\omega_{2}\right)
$$

The downconversion event can happen at any point along the propagation of the pump pulse through the nonlinear crystal, and the created two-photon state $|\Psi\rangle$ is, in general, a superposition of all photon states $\left|\omega_{1}, \mathbf{k}_{1} ; \omega_{2}, \mathbf{k}_{2}\right\rangle$, for which Eqs. (1) and (2) are both satisfied. The entangled two-photon state then reads

$$
|\Psi\rangle=\int d \mathbf{k}_{1} \int d \mathbf{k}_{2} \Psi\left(\mathbf{k}_{1}, \mathbf{k}_{2}\right)\left|\omega_{1}, \mathbf{k}_{1} ; \omega_{2}, \mathbf{k}_{2}\right\rangle
$$

In the following, we neglect the spatial variation of the two-photon wavefunction (for a review, see e.g. Ref. ${ }^{31}$ ), and assume that the spatial propagation direction is selected, e.g., by small slits ${ }^{24}$ or by collinear downconversion.

A typical representation of the resulting wavefunction in frequency domain $\Psi\left(\omega_{1}, \omega_{2}\right)$ is sketched in the left panel of Fig. 1(b), which shows the absolute value of the amplitude vs. the photon frequencies $\omega_{1}$ and $\omega_{2}$. The width along the diagonal, $\Delta \omega_{+}$, is determined by the pump bandwidth, due to energy conservation in Eq. (1). The anti-diagonal width $\Delta \omega_{-}$ 
is determined by the momentum phase matching, Eq. (2), and can be much broader than $\Delta \omega_{+}$

The corresponding time domain wavefunction $\tilde{\Psi}\left(t_{1}, t_{2}\right)$ obtained by a double Fourier transform is shown in the right panel of Fig. 1(b). The small uncertainty $\Delta \omega_{+}$translates into a large uncertainty $\Delta t_{+}$. Hence, the photon pair arrival time is limited by the pump duration. Yet the large uncertainty $\Delta \omega_{-}$implies a strong correlation of the photon arrival times $\Delta t_{-}$. Once the first photon is absorbed e.g. in a detector, its twin must arrive within a very narrow time window, known as the entanglement time $T$. When $\Delta \omega_{+}=0$, this time window is solely determined by the group velocity mismatch within the crystal, and can be controlled by the crystal length, ${ }^{32}$ as we shall examine below.

Taking only the two emerging propagation directions into account, a typical form for such an entangled state is given by the twin photon state ${ }^{24}$

$$
\begin{aligned}
\left|\Psi_{\text {twin }}\right\rangle=\frac{1}{\sqrt{\mathcal{N}}} \int d \omega_{1} \int d \omega_{2} \exp \left(-\left(\omega_{1}+\omega_{2}-\omega_{p}\right)^{2} / \Delta \omega_{+}^{2}\right) \\
\quad \times \operatorname{sinc}\left(\frac{\ell}{2 \pi}\left(k_{1}\left(\omega_{1}\right)+k_{2}\left(\omega_{2}\right)-k_{p}\left(\omega_{1}+\omega_{2}\right)\right)\right)\left|\omega_{1}, \omega_{2}\right\rangle,
\end{aligned}
$$

where $\ell$ is the crystal length, and the normalization constant $\mathcal{N}=\Delta \omega_{+} \pi^{3 / 2} /(2 T)$. The first exponential stems from the pump pulse bandwidth, and the sinc-function ${ }^{33}$ arises from the phase matching inside the crystal, and gives rise to the entanglement time $T .{ }^{32}$

Strong correlations in both the time and frequency domains are a hallmark of entanglement in the pure quantum state $|\Psi\rangle$. If we were to replace the superposition state by a classically correlated mixture of states, where the density matrix looks like $\varrho \sim \sum_{\omega_{1}, \omega_{2}} p_{\omega_{1}, \omega_{2}}\left|\omega_{1}, \omega_{2}\right\rangle\left\langle\omega_{1}, \omega_{2}\right|$, we would only reproduce the frequency but not the time correlations (see e.g. Ref. ${ }^{34}$ ).

\subsection{Photon counting}

We now consider a setup, where the entangled photons can be detected individually. According to the standard theory of photodetection, the average number of photons in beam $i$ 
is given by ${ }^{28}$

$$
\begin{aligned}
n_{i} & =\eta \int_{-t_{w} / 2}^{t_{w} / 2} d \tau\left\langle\Psi\left|a_{i}^{\dagger}(\tau) a_{i}(\tau)\right| \Psi\right\rangle \\
& \equiv \eta t_{w} A_{d} \Phi,
\end{aligned}
$$

where $t_{w}$ denotes the detection window, $\eta$ the detector efficiency, $A_{d}$ the detector area, and $a_{i}(\tau)$ the photon annihilation operator at time $\tau$, with the commutation relation $\left[a_{i}(t), a_{i}^{\dagger}\left(t^{\prime}\right)\right]=$ $\delta\left(t-t^{\prime}\right)$. The expectation value is taken with respect to the photon field which in our case is the entangled two-photon state $|\Psi\rangle$, e.g. Eq. (4). In the second line, we have defined the photon flux density $\Phi$ - the number of detector clicks per unit time and area. The prefactor $\eta A_{d}$ can be interpreted as the detector's single photon absorption cross section associated with $|\Psi\rangle$. In the case of the twin state (4), if the time window $t_{w}$ is much longer than the temporal length of the photon wavepacket but small enough such that only a single photon pair is measured, we obtain $n_{i} \simeq \eta A_{d} / A_{q}$, where $A_{q}$ is the quantisation area (the beam area perpendicular to its propagation direction $\left.{ }^{35}\right)$, and hence $\Phi \simeq 1 /\left(t_{w} A_{q}\right)$.

The detection probability of two photons from the two beams, where the second photon is detected with a time delay $t$, is given by

$$
\begin{aligned}
G^{(2)}(t) & =\eta^{2} \int_{-t_{w} / 2}^{t_{w} / 2} d \tau\left\langle\Psi\left|a_{1}^{\dagger}(\tau) a_{2}^{\dagger}(\tau+t) a_{2}(\tau+t) a_{1}(\tau)\right| \Psi\right\rangle \\
& \equiv \eta^{2} f(t) t_{w} A_{d 1} A_{d 2} \Phi+\ldots
\end{aligned}
$$

This coincidence signal peaks at $t=0$, with a temporal width determined by $\Delta t_{-}$(see Fig. 1(b)). Since the expectation value is taken with respect to the same two-photon state $|\Psi\rangle$ as in Eq. (5), it also depends linearly the photon flux density $\Phi$ to leading order. This is again shown in the second line, where we write the coincidence signal as the product of the delay dependence $f(t)$, the detector efficiency $\eta^{2}$, the detection window $t_{w}$ and the two detector areas $A_{d 1}$ and $A_{d 2}$. Hence, at zero delay $t=0, \eta^{2} A_{d 1} A_{d 2}$ can be seen as 
the detector's entangled two-photon absorption cross section. When the photon flux is high, different photon pairs can overlap temporally, creating an incoherent background of uncorrelated coincidences which scales quadratically with $\Phi$ and only depends on the the temporal width $\Delta t_{+}$in Fig. $1(\mathrm{~b})$.

\section{Entangled two-photon absorption}
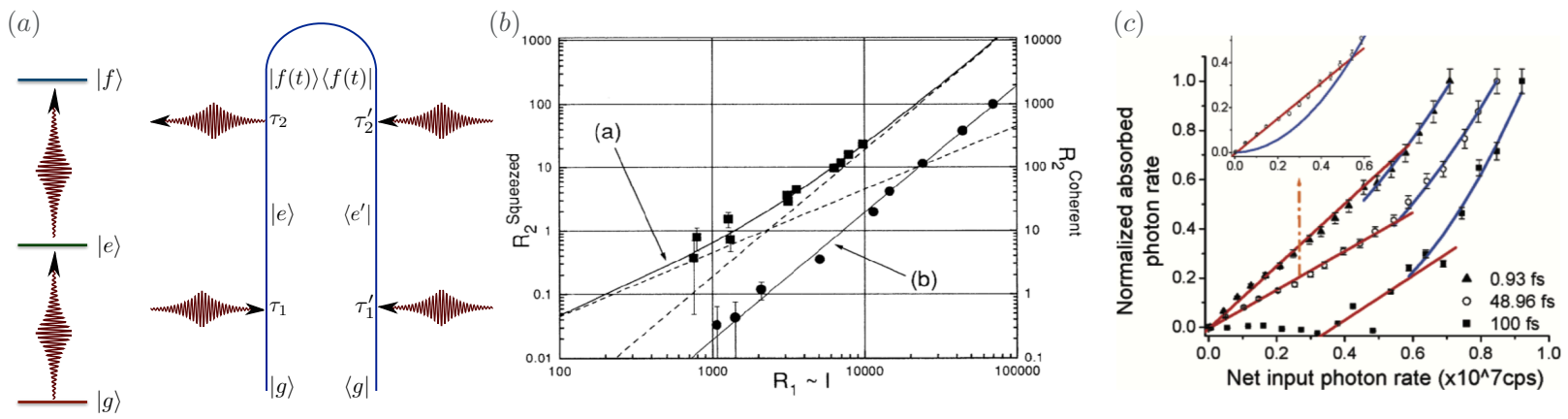

Figure 2: (a) The level scheme and the Feynman diagram for the leading contribution to the final state population, Eq. (9). For an introduction to the diagrammatic technique, see e.g. ${ }^{36,37}$. Linear scaling of the TPA rate in (b) an atomic sample, ${ }^{16}$ and (c) in a molecular sample. ${ }^{19}$ Reproduced with permission from ref., ${ }^{16}$ Copyright 1995 American Physical Society, and ref., ${ }^{19}$ Copyright 2006 American Chemical Society.

Two-photon absorption (TPA) experiments with entangled photons were first carried in atomic, ${ }^{16-18}$ and more recently, in molecular samples. ${ }^{19-22,38,39}$ These simplest nonlinear optical experiments detect the probability of excitation of the final state $f$, where $\omega_{1}+\omega_{2}=\omega_{f g}$ (see the level scheme in Fig. 2(a)). This probability can be detected e.g by characteristic fluorescence. Formally, a TPA experiment is very similar to a photon coincidence measurement, when the two detectors are replaced by the sample system. Hence, much of what was discussed in the previous section applies to TPA as well. The TPA signal $R_{\text {TPA }}\left(s^{-1}\right)$ contains two contributions

$$
R_{\mathrm{TPA}}=\sigma_{e} \Phi+\delta_{r} \Phi^{2}
$$


where the prefactors are the correlated absorption cross section $\sigma_{e}$, and the classical absorption cross section $\delta_{r}$, respectively. The first term stems from the absorption of an entangled pair, and is hence linear in the photon flux, and the second quadratic contribution originates from the absorption of two uncorrelated photons from different photon pairs, so that the effect of entanglement is eliminated, and the standard classical quadratic scaling is recovered. A TPA measurement with classical laser light would also measure $\delta_{r}$. This scaling was detected in both atomic and molecular samples, as shown in Fig. 2(b) and (c), respectively. In both cases, the signal can only be fitted accurately by including a linear and a quadratic contribution. The crossover between the linear and the quadratic regime depends on properties of the entangled states. As pointed out in Ref., ${ }^{18}$ the maximal photon flux at which the light can be considered as composed of individual photon pairs is given by the photon pair bandwidth. If this flux is exceeded, different pairs start to overlap. Hence, the larger the photon bandwidth, the better the linear regime can be observed. In broadband downconversion of a narrowband pump pulse, where the two-photon wavefunction is sketched in Fig. 1(b), this means that a short entanglement time $T$ (strong entanglement) is beneficial.

The entangled two-photon absorption cross section can be calculated as ${ }^{24,36,40}$

$$
\sigma_{e}=\frac{A_{q}^{2}}{A_{e}} \int_{-\infty}^{\infty} d t \frac{d}{d t} p_{f}(t)
$$

where $A_{e}$ is the entanglement area (the area perpendicular to the propagation direction in which the entangled pair was created), and $p_{f}(t)$ denotes the population in the final state at time $t$. Typically, it is equivalent to calculating $p_{f}(t)$, and then taking the limit $t \rightarrow \infty$. In the limit $\Delta \omega_{+}=0$ (i.e. in the cw pump limit), one simply calculates the steady-state population $p_{f}$.

The corresponding Feynman diagram for the population in $f$, shown in Fig. 2(a), trans- 
lates into the following expression

$$
\begin{aligned}
p_{f}(t)=\left(-\frac{i}{\hbar}\right)^{4} \int_{t_{0}}^{t} d \tau_{2} \int_{t_{0}}^{\tau_{2}} d \tau_{1} \int_{t_{0}}^{t} d \tau_{2}^{\prime} \int_{t_{0}}^{\tau_{2}^{\prime}} d \tau_{1}^{\prime}\left\langle V\left(\tau_{1}^{\prime}\right) V\left(\tau_{2}^{\prime}\right) V^{\dagger}\left(\tau_{2}\right) V^{\dagger}\left(\tau_{1}\right)\right\rangle \\
\times\left\langle\Psi\left|E^{\dagger}\left(\tau_{1}^{\prime}\right) E^{\dagger}\left(\tau_{2}^{\prime}\right) E\left(\tau_{2}\right) E\left(\tau_{1}\right)\right| \Psi\right\rangle
\end{aligned}
$$

where $V$ and $V^{\dagger}$ denote the positive- (negative-) frequency component of the dipole operator, respectively. $V$ destroys an excitation, whereas $V^{\dagger}$ creates one. The expectation value $\langle\ldots\rangle$ is taken with respect to the initial density matrix of the sample. Likewise, $E$ and $E^{\dagger}$ denote the positive- (negative-) frequency components of the electric field operator. Eq. (9) can be interpreted as follows: the spectroscopic information is contained in $\left\langle V V V^{\dagger} V^{\dagger}\right\rangle$, and the field correlation $\left\langle E^{\dagger} E^{\dagger} E E\right\rangle$ serves as an observation window.

Eq. (9) is a time-ordered convolution of the matter and the field correlation functions, and disentangling the impact of quantum correlations and fluctuations, and of quantum dynamics within the material is not an easy task. It implies that the entangled two-photon absorption cross sections defined in Eqs. (7) and (8) depend on the incident photonic state,

$$
\sigma_{e}=\sigma_{e}(\Psi)
$$

and quantum correlations influence the absorption cross section. In contrast, the semiclassical limit of Eq. (9) is obtained by replacing the field correlation function in the second line by a product of classical field amplitudes. It is then possible to define the classical cross section $\delta_{r}$ without reference to the classical field amplitude, and by only evaluating the material correlation function $\left\langle V V V^{\dagger} V^{\dagger}\right\rangle$.

A clear picture for the impact of quantum correlations emerges for a monochromatic pump driving the downconversion process $\left(\Delta \omega_{+}=0\right)$, where the steady state $p_{f}$ is given by the product of two transition amplitudes. ${ }^{24,26,40,41}$ Using Eq. (4) in this limit, we obtain 
$p_{f}=\left|T_{f g}\right|^{2}$ with the transition amplitudes

$$
T_{f g} \propto \sum_{e} \frac{\mu_{g e} \mu_{e f}}{\xi_{p f}} \frac{1}{\sqrt{T}}\left(\frac{e^{i \xi_{1 e} T}-1}{\xi_{1 e}}+\frac{e^{i \xi_{2 e} T}-1}{\xi_{2 e}}\right) .
$$

Here, the summation runs over all intermediate states $e, \mu_{g e}$ and $\mu_{e f}$ denote the dipole moments of the $g \rightarrow e$ and $e \rightarrow f$ transitions, respectively. $\xi_{i e}=\omega_{i}^{(0)}-\omega_{e g}+i \gamma_{e}$ is a complex phase which is composed of the detuning between the central frequency of the $i$-th photon's wavepacket $\omega_{i}^{(0)}$ from the intermediate state's frequency $\omega_{e g}$, and the excited state lifetime $\gamma_{e}$. Similarly, $\xi_{p f}=\omega_{p}-\omega_{f g}+i \gamma_{f}$ is composed of the detuning between pump frequency and the final state, and the final state lifetime $\gamma_{f}$. The first factor in Eq. (11) is simply the normal Lorentzian resonance of the two-photon transition, $\sim 1 / \xi_{p f}$. The second term combines the entanglement time $T$ with the single-photon transition resonances $\xi_{i e}$ : The second photon must arrive within the entanglement time $T$, thus broadening the resonance of the $g \rightarrow e$ transition like a sinc-function. When $T^{-1} \gg\left|\omega_{i}^{(0)}-\omega_{e g}\right|$ and $T^{-1} \gg \gamma_{e}$, we can expand the exponential to obtain $T_{f g} \propto \sqrt{T}$ (hence $\sigma_{e} \propto T$ ), and the two-photon transition no longer depends on the detuning $\omega_{i}^{(0)}-\omega_{e}$ between the photon and intermediate state frequencies, since the photon bandwidth is much larger. In the opposite limit, when $T^{-1} \ll \gamma_{e}$, the exponential vanishes due to the finite $\gamma_{e}$, and the remaining expression becomes a normal single-photon resonance. We thus recover the classical two-photon absorption resonance, and $T_{f g} \propto 1 / \sqrt{T}$.

As discussed in the previous section, in the high photon-flux regime, when different photon pairs overlap temporally, incoherent contributions have to be added, ${ }^{42,43}$ giving rise to the second term $\delta_{r} \Phi^{2}$ in Eq. (7), recovering an essentially classical regime in which quantum correlations do not affect the signal.

One intriguing question originallly raised in ${ }^{44}$ concerns the possibility of a collective response of many sample molecules: Can entanglement be employed to induce collective resonances in the optical response, where the signal would be created by two or more molecules? 
It turns out, however, that two-photon excitation of a collection of noninteracting two-level atoms does not result in collective resonance due to destructive interference of excitation pathways. Nevertheless, they may be observed in photon statistics (Hanbury-Brown-Twiss) measurements through the attenuation of two-time intensity correlations. ${ }^{45}$

\section{Population dynamics}

In the case of a multilevel system such as a molecular photosynthetic aggregate, the timeenergy entanglement can control the excited state distributions, and suppress energy relaxation, ${ }^{41,46}$ or control vibronic states in a molecule. ${ }^{47,48}$ As a minimal example to illustrate this, we depict simulations of a five-level toy model from Ref. ${ }^{29}$ in the left panel of Fig. 3(a), where the intermediate states $\left|e_{1}\right\rangle$ and $\left|e_{2}\right\rangle$ undergo rapid incoherent relaxation $\left|e_{2}\right\rangle \rightarrow\left|e_{1}\right\rangle$. The goal is to suppress relaxation, which always drives the population towards the same low-energy state. This is possible with short pulses in classical spectroscopy. At the same time, we want to keep the spectral resolution, which would require narrow bandwidth pulses. Both goals can be achieved with entangled photons. In Fig. 3(a), the relative population in $\left|f_{1}\right\rangle$ and $\left|f_{2}\right\rangle$ (the ratio of population deposited in either state) after excitation by entangled photons is contrasted with the excitation by classical laser pulses (shown as a function of twice their center frequency $\left.\omega_{0}\right)$. The classical pulses have bandwidths $\Delta \omega_{+}$or $\Delta \omega_{-}$, respectively, such that they reproduce either the diagonal or the anti-diagonal of the entangled photon two-photon wavefunction in Fig. 1. This means, these pulses reproduce either the frequency resolution or the time resolution in the arrival time of the entangled photon pairs. While entangled photons can be designed to move almost the entire population into one of the two states, this is not possible with laser pulses. Either the broad bandwidth of temporally short pulses inhibits a targeted excitation, or, when using narrow bandwidth light, the intermediate relaxation drives the e-population towards a thermal state, thus creating the similar $f$-state distributions regardless of their central laser frequency. The reason for 
(a)
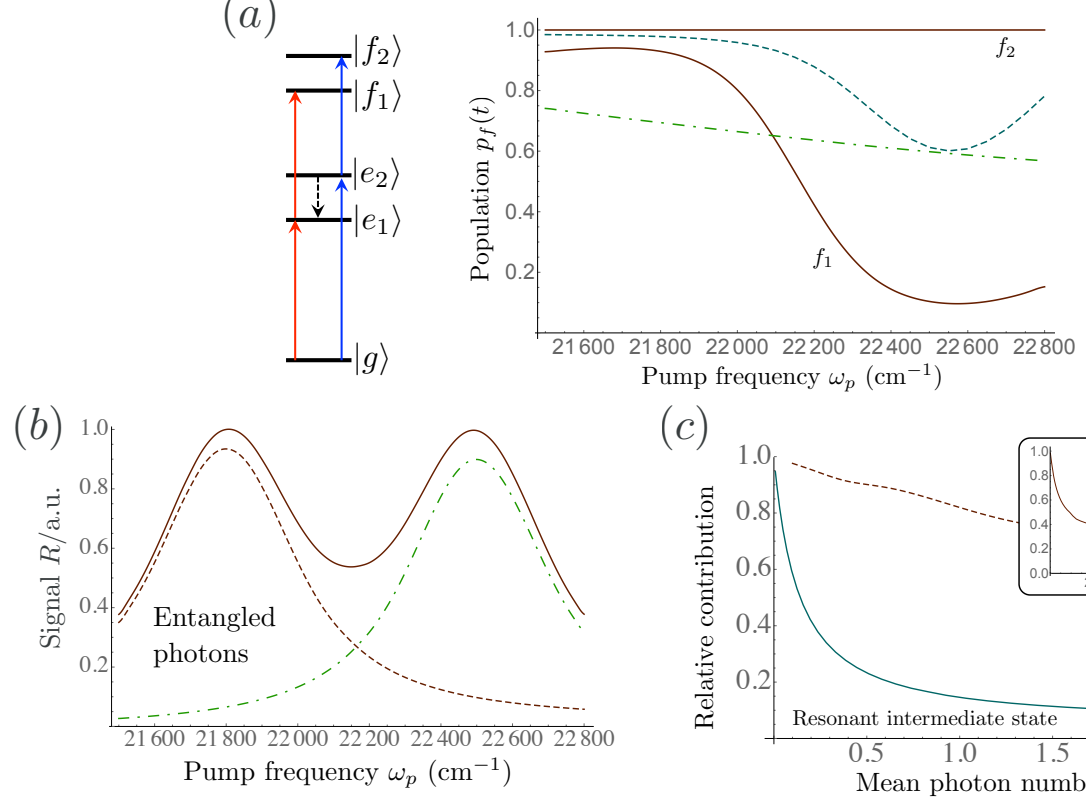

(c)
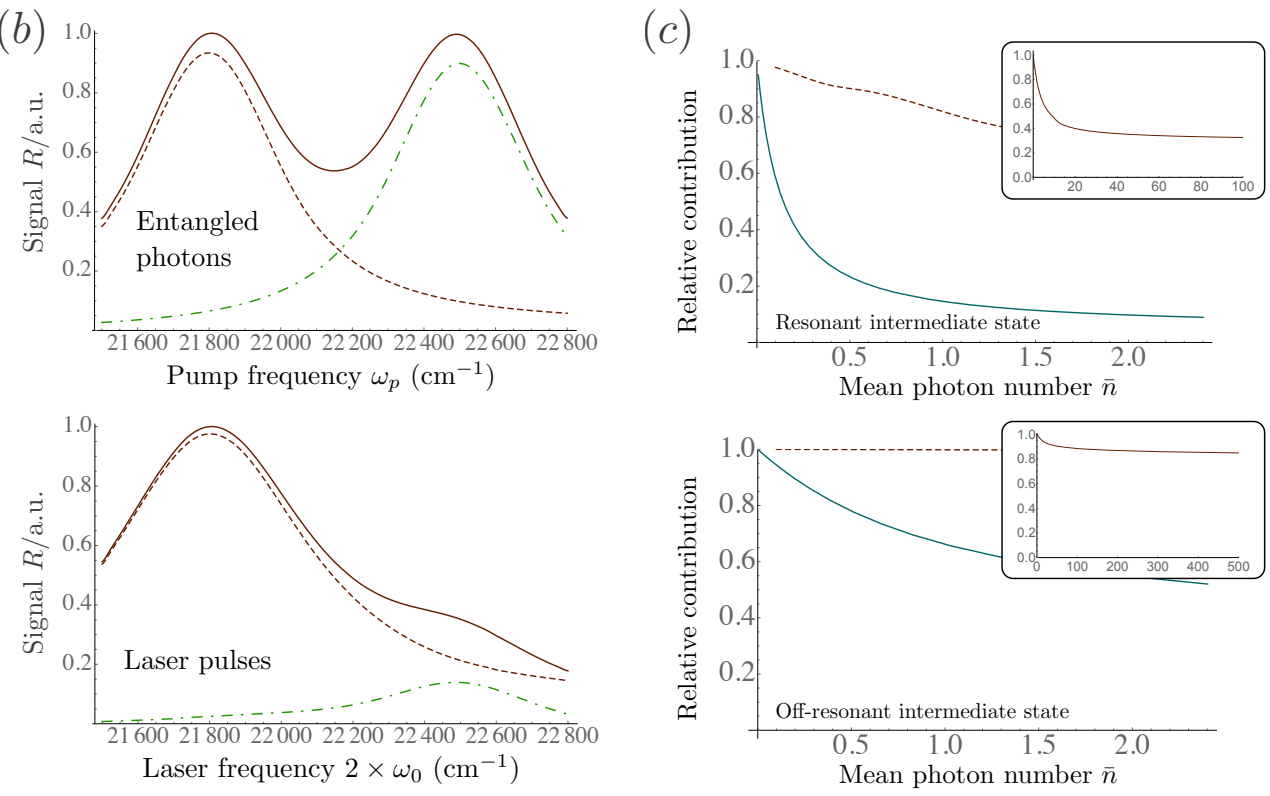

Figure 3: (a) 5-level model system and the $f$-state population excited by entangled photons (solid lines), and by classical pulses matching either the bandwidth $\Delta \omega_{+}$(dashed line) or $\Delta \omega_{-}$ (dot-dashed line) in Fig. 1. Each line indicates the ratio of population in $f_{1}$ at a given pump frequency. E.g. at $\omega_{p}=21800 \mathrm{~cm}^{-1}$, entangled photons excite ca. $95 \% f_{1}$, and $5 \% f_{2}$, while short classical pulses (dot-dashed) excite ca. $70 \% f_{1}$ and $30 \% f_{2}$. (b) The fluorescence signal collected from the populations in (a). Top: entangled photons. Bottom: classical pulses with bandwidth $\Delta \omega_{+}$. The dashed, and dot-dashed lines indicate the signal created by emission from the $f_{1}$ or $f_{2}$, respectively. (c) Relative contributions of the coherent and the incoherent absorption of photons in a three-level system, where the intermediate state is either resonant (solid blue line) or off-resonant (dashed red line), are plotted vs. the mean photon number of the incoming light field. The insets show extrapolations of the signal with off-resonant intermediate state to larger photon numbers.

Reproduced with permission from Ref. ${ }^{29}$ Copyright 2016 American Physical Society

this advantage of entangled photons can be traced back to the classical Fourier uncertainty, which has to be satisfied for every single absorption event. Consequently, for a two-photon 
transition, it restricts the simultaneous time and frequency resolution, ${ }^{28,46}$

$$
\Delta \omega_{c l} \times \Delta t_{c l} \geq 1
$$

where $\Delta \omega_{c l}$ denotes the uncertainty in the frequency resolution, and $\Delta t_{c l}$ the uncertainty in the time delay between the two absorption events. Thus, the larger we make the pulse bandwidth to minimize $\Delta t_{c l}$, the larger $\Delta \omega_{c l}$ becomes, and we lose in frequency resolution. In two-photon absorption of entangled photons, the photonic and the material systems are entangled in between absorption events. The uncertainties are determined by frequency width $\Delta \omega_{+}$and the time delay $\Delta t_{-}$in Fig. 1(b). These are not Fourier conjugates, and can thus violate the inequality (12).

The TPA signal based on the distributions in Fig. 3(a) is shown in Fig. 3(b). The entangled photon signal has two resonances of equal magnitude, each can be attributed to the signal from one of the $f$-states, respectively (as indicated by the dashed lines below). The signal created by laser pulses below only features one strong resonance, with the other one merely visible as a weak shoulder. It is impossible to efficiently excite $\left|f_{2}\right\rangle$, and hence it can be difficult to detect this state. So on top of the lack of control over the excited $f$-state distribution, the overall excitation efficiency is also dramatically reduced. Entangled photons offer a distinct advantage in such a situation stemming from the simultaneous time and frequency correlations of the entangled quantum state of light. It cannot be reproduced by classical correlations.

This advantage of entangled photons can persist even for high photon numbers. In Fig. 3(c), we show the relative contribution of the coherent excitation [the linear term in Eq. (7)] and the incoherent excitation (the quadratic term) as a function of the mean photon number $\bar{n}$ in the initial photonic state in a three-level system. If the intermediate state is off-resonant, the quantum correlations are essential for the efficient excitation, and hence the coherent contribution can dominate the signal up to very large photon numbers. In resonant 
excitation, they are not as important, and the coherent signal is more easily degraded.
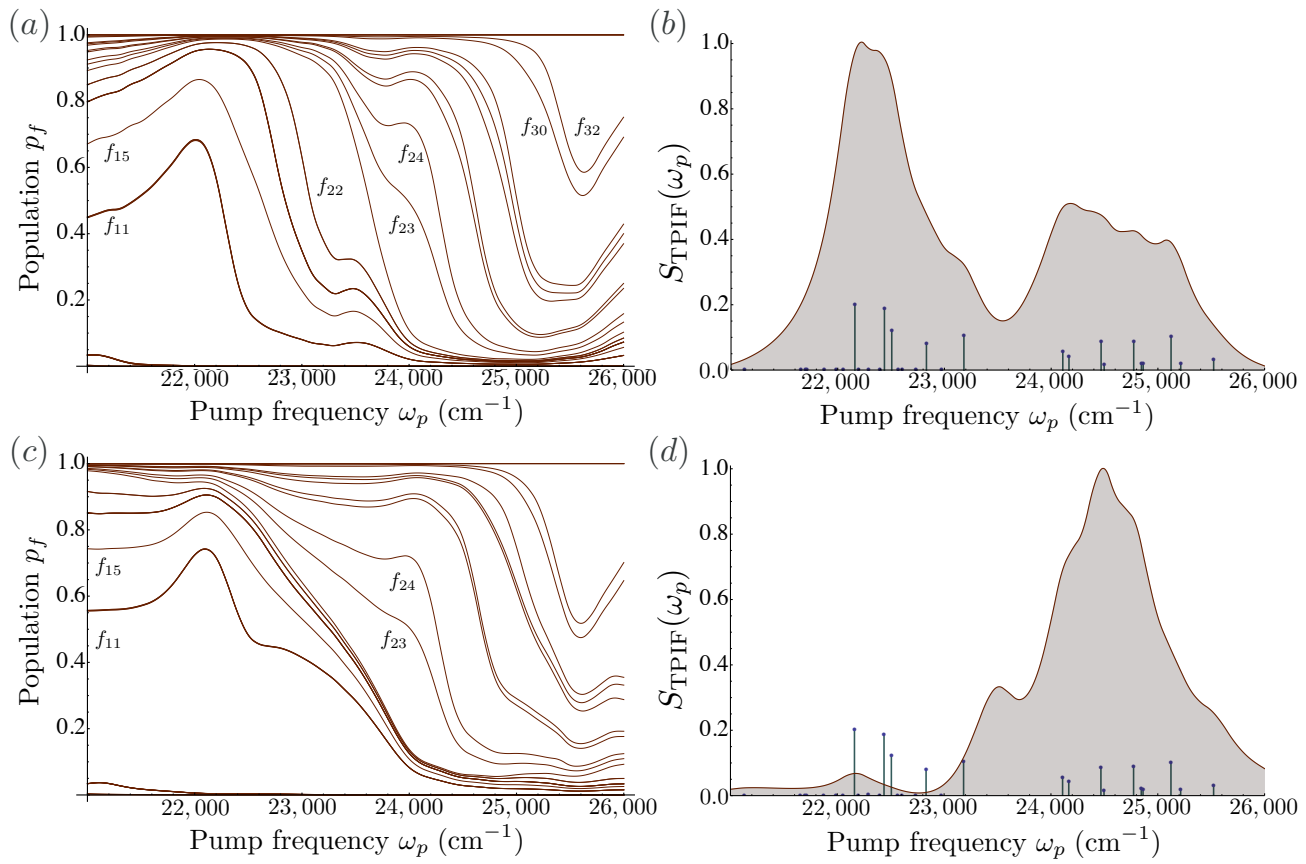

Figure 4: (a) Relative doubly excited $f$-state population distribution in a model of the bacterial reaction center of $\mathrm{B}$. viridis excited by entangled photons with entanglement time $T=10$ fs. The interpretation of the figure is identical to Fig. 3(a). (b) The two-photon induced fluorescence (TPIF) signal of the distribution in (a). (c) Same as (a) with entanglement time $T=100 \mathrm{fs}$. (d) TPIF signal of the distribution in (c). Adapted from Ref. ${ }^{49}$ Copyright 2016 Springer Publishing.

The unique properties of entangled photons outlined above also persist in realistic models of photosynthetic complexes. In Fig. 4 we present simulations of entangled two-photon absorption in a bacterial reaction center with six chlorophyll molecules, described by a tightbinding model with 12 single-exciton, and 41 two-exciton states. As in Fig. 3(a), the left panel in the top row shows the relative excitation of the individual $f$-states created by strongly entangled photon pairs with entanglement time $T=10 \mathrm{fs}$, and the right panel shows the twophoton induced fluorescence signal (which contains the same information as the TPA signal) created by these $f$-state distributions. The TPIF signal peaks whenever the pump frequency is on resonance with a two-exciton energy. The bottom row depicts the same simulation with entangled pairs with $T=100 \mathrm{fs}$. The intermediate energy relaxation has greatly reshuffled the population distributions, and the emerging TPIF signal has changed almost completely. 
Thus, it is possible to detect ultrafast relaxation processes using entangled photons without the need for phase-stabilized detection or ultrafast laser pulses.

\section{Conclusions and Outlook}

We have reviewed how the two-photon absorption signal can be manipulated by entangled photon pairs. The entangled absorption cross section depends on the initial photonic quantum state, and may not be defined without reference to this state. Instead, the signal depends on a multidimensional convolution of the sample and field correlation functions. This property was not covered in this review, yet it opens the possibility of shaping two-photon states in order to maximize the cross section. ${ }^{50-53}$

If the sample consists of a multilevel system with complex internal dynamics, the convolution of field and matter response strongly affects the excitation pathways, as well as the time and frequency resolution of the information that can be extracted from the sample. It may be used to suppress population transport, control excited state distributions, and thereby enhance otherwise very weak signals, or examine ultrafast relaxation dynamics.

The physics reviewed in this paper could be combined with established methods to extract phase-dependent multidimensional signals, as proposed in. ${ }^{28}$ These developments promise to establish entangled photon spectroscopy a new, highly versatile tool for nonlinear spectroscopy in the near future.

\section{Biographical Information}

Frank Schlawin completed his undergraduate studies in physics and received his P.h.D. at the University of Freiburg, Germany. Since 2015, he works as a postdoctoral researcher at the University of Oxford, and since 2017 as a College Lecturer at Keble College, Oxford. His research focuses on quantum optics, nonlinear spectroscopy with quantum light, quantum transport, driven many-body systems and cavity quantum electrodynamics. 
Konstantin E. Dorfman completed his B.S. degree in Physics from Nizhny Novgorod State University, Russia, and his Ph.D. degree at Texas A\&M University, where he investigated many-body fluctuations of Bose-Einstein condensates. After positions as a joint postdoctoral fellow at Princeton University and Texas A\&M University (2010 - 2012), and a postdoctoral research scholar at the University of California, Irvine, from 2012 to 2015 he became staff scientist at the Singapore Agency for Science, Technology and Research. He

is now appointed as a thousand young talents professor of physics at State Key Laboratory of Precision Spectroscopy, East China Normal University, Shanghai. His scientific interests include atomic, molecular, optical, and chemical physics, energy and charge transport processes, quantum optics and electronics, X-ray optics, relativistic and Terahertz physics, statistical mechanics, many-body theory, quantum photovoltaics, and semiconductor physics.

Shaul Mukamel, currently Distinguished Professor of Chemistry and Physics and Astronomy at the University of California, Irvine, received his Ph.D. in 1976 from Tel Aviv University. Following postdoctoral appointments at MIT and the University of California, Berkeley, he has held faculty positions at Rice University, the Weizmann Institute, and the University of Rochester. His research interests span all areas of ultrafast multidimensional spectroscopy from the infrared to the $\mathrm{X}$ ray regimes, excitons in chromophore aggregates and molecular electrodynamics.

\section{Acknowledgement}

F.S. acknowledges funding from the European Research Council under the European Union's Seventh Framework Programme (FP7/2007-2013) Grant Agreement No. 319286 Q-MAC. S.M gratefully acknowledges the support of the National Science Foundation (grant CHE1663822) and of the Chemical Sciences, Geosciences, and Biosciences division, Office of Basic Energy Sciences, Office of Science, U.S. Department of Energy through award No. DE-FG0204ER15571. K.E.D. is supported by the Zijiang Endowed Young Scholar Fund and Overseas 
Expertise Introduction Project for Discipline Innovation (111 Project, B12024).

\section{References}

1 Einstein, A.; Podolsky, B.; Rosen, N. Can Quantum-Mechanical Description of Physical Reality Be Considered Complete? Phys. Rev. 1935, 47, 777-780.

2 Bell, J. S. In John S. Bell on The Foundations of Quantum Mechanics; Bell, M., Gottfried, K., Veltman, M., Eds.; World Scientific Publishing Co, Pte. Ltd.: Singapore, SE Asia, 2001; pp 7-12.

3 Aspect, A.; Grangier, P.; Roger, G. Experimental Tests of Realistic Local Theories via Bell's Theorem. Phys. Rev. Lett. 1981, 47, 460-463.

4 Aspect, A.; Grangier, P.; Roger, G. Experimental Realization of Einstein-PodolskyRosen-Bohm Gedankenexperiment: A New Violation of Bell's Inequalities. Phys. Rev. Lett. 1982, 49, 91-94.

5 Aspect, A.; Dalibard, J.; Roger, G. Experimental Test of Bell's Inequalities Using TimeVarying Analyzers. Phys. Rev. Lett. 1982, 49, 1804-1807.

6 Gisin, N.; Ribordy, G.; Tittel, W.; Zbinden, H. Quantum cryptography. Rev. Mod. Phys. 2002, 7/, 145-195.

7 Pittman, T. B.; Shih, Y. H.; Strekalov, D. V.; Sergienko, A. V. Optical imaging by means of two-photon quantum entanglement. Phys. Rev. A 1995, 52, R3429-R3432.

8 D'Angelo, M.; Chekhova, M. V.; Shih, Y. Two-Photon Diffraction and Quantum Lithography. Phys. Rev. Lett. 2001, 87, 013602.

9 Nasr, M. B.; Saleh, B. E. A.; Sergienko, A. V.; Teich, M. C. Demonstration of DispersionCanceled Quantum-Optical Coherence Tomography. Phys. Rev. Lett. 2003, 91, 083601. 
10 Resch, K.; Puvanathasan, P.; Lundeen, J.; Mitchell, M.; Bizheva, K. Classical dispersioncancellation interferometry. Opt. Express 2007, 15, 8797-8804.

11 Kaltenbaek, R.; Lavoie, J.; Biggerstaff, D. N.; Resch, K. J. Nature Phys. 2008, 4, 864.

12 Lavoie, J.; Kaltenbaek, R.; Resch, K. J. Quantum-optical coherence tomography with classical light. Opt. Express 2009, 17, 3818-3826.

$13 \mathrm{Ou}, \mathrm{Z}$. Y.; Hong, C. K.; Mandel, L. Coherence properties of squeezed light and the degree of squeezing. J. Opt. Soc. Am. B 1987, 4, 1574-1587.

14 Gea-Banacloche, J. Two-photon absorption of nonclassical light. Phys. Rev. Lett. 1989, 62, 1603-1606.

15 Javanainen, J.; Gould, P. L. Linear intensity dependence of a two-photon transition rate. Phys. Rev. A 1990, 41, 5088-5091.

16 Georgiades, N. P.; Polzik, E. S.; Edamatsu, K.; Kimble, H. J.; Parkins, A. S. Nonclassical Excitation for Atoms in a Squeezed Vacuum. Phys. Rev. Lett. 1995, 75, 3426-3429.

17 Dayan, B.; Pe'er, A.; Friesem, A. A.; Silberberg, Y. Two Photon Absorption and Coherent Control with Broadband Down-Converted Light. Phys. Rev. Lett. 2004, 93, 023005.

18 Dayan, B.; Pe'er, A.; Friesem, A. A.; Silberberg, Y. Nonlinear Interactions with an Ultrahigh Flux of Broadband Entangled Photons. Phys. Rev. Lett. 2005, 94, 043602.

19 Lee, D.-I.; Goodson, T. Entangled Photon Absorption in an Organic Porphyrin Dendrimer. J. Phys. Chem. B 2006, 110, 25582-25585.

20 Harpham, M. R.; Suzer, O.; Ma, C.-Q.; Bäuerle, P.; Goodson, T. Thiophene Dendrimers as Entangled Photon Sensor Materials. J. Am. Chem. Soc. 2009, 131, 973-979. 
21 Guzman, A. R.; Harpham, M. R.; Suzer, O.; Haley, M. M.; Goodson, T. G. Spatial Control of Entangled Two-Photon Absorption with Organic Chromophores. J. Am. Chem. Soc. 2010, 132, 7840-7841.

22 Upton, L.; Harpham, M.; Suzer, O.; Richter, M.; Mukamel, S.; Goodson, T. Optically Excited Entangled States in Organic Molecules Illuminate the Dark. J. Phys. Chem. Lett. 2013, 4, 2046-2052.

23 Saleh, B. E. A.; Jost, B. M.; Fei, H.-B.; Teich, M. C. Entangled-Photon Virtual-State Spectroscopy. Phys. Rev. Lett. 1998, 80, 3483-3486.

24 Fei, H.-B.; Jost, B. M.; Popescu, S.; Saleh, B. E. A.; Teich, M. C. Entanglement-Induced Two-Photon Transparency. Phys. Rev. Lett. 1997, 78, 1679-1682.

25 Roslyak, O.; Marx, C. A.; Mukamel, S. Nonlinear spectroscopy with entangled photons: Manipulating quantum pathways of matter. Phys. Rev. A 2009, 79, 033832.

26 Roslyak, O.; Mukamel, S. Multidimensional pump-probe spectroscopy with entangled twin-photon states. Phys. Rev. A 2009, 79, 063409.

27 Richter, M.; Mukamel, S. Ultrafast double-quantum-coherence spectroscopy of excitons with entangled photons. Phys. Rev. A 2010, 82, 013820.

28 Raymer, M. G.; Marcus, A. H.; Widom, J. R.; Vitullo, D. L. P. Entangled Photon-Pair Two-Dimensional Fluorescence Spectroscopy (EPP-2DFS). J. Phys. Chem. B 2013, 117, 15559-15575.

29 Dorfman, K. E.; Schlawin, F.; Mukamel, S. Nonlinear optical signals and spectroscopy with quantum light. Rev. Mod. Phys. 2016, 88, 045008.

30 Schlawin, F. Entangled photon spectroscopy. J. Phys. B: At. Mol. Opt. 2017, 50, 203001.

31 Walborn, S.; Monken, C.; Pádua, S.; Ribeiro, P. S. Spatial correlations in parametric down-conversion. Phys. Rep. 2010, 495, 87 - 139. 
32 Joobeur, A.; Saleh, B. E. A.; Larchuk, T. S.; Teich, M. C. Coherence properties of entangled light beams generated by parametric down-conversion: Theory and experiment. Phys. Rev. A 1996, 53, 4360-4371.

$33 \operatorname{sinc}(x)=\sin (x) / x$.

34 Lerch, S.; Stefanov, A. Observing the transition from quantum to classical energy correlations with photon pairs. Commun. Phys. 2018, 1, 26.

35 Loudon, R. The Quantum Theory of Light; Oxford University Press: Oxford, UK, 2000.

36 Mukamel, S.; Rahav, S. In Advances in Atomic, Molecular, and Optical Physics; Arimondo, E., Berman, P., Lin, C., Eds.; Advances In Atomic, Molecular, and Optical Physics; Academic Press, 2010; Vol. 59; pp 223 - 263.

37 Hansen, T.; Pullerits, T. Nonlinear response theory on the Keldysh contour. J. Phys. B: At. Mol. Opt. 2012, 45, 154014.

38 Villabona-Monsalve, J. P.; Calderon-Losada, O.; Nunez Portela, M.; Valencia, A. Entangled Two Photon Absorption Cross Section on the $808 \mathrm{~nm}$ Region for the Common Dyes Zinc Tetraphenylporphyrin and Rhodamine B. J. Phys. Chem. A 2017, 121, 7869-7875, PMID: 28933852.

39 Varnavski, O.; Pinsky, B.; Goodson, T. Entangled Photon Excited Fluorescence in Organic Materials: An Ultrafast Coincidence Detector. J. Phys. Chem. Lett. 2017, 8, 388393, PMID: 28029793.

40 Lissandrin, F.; Saleh, B. E. A.; Sergienko, A. V.; Teich, M. C. Quantum theory of entangled-photon photoemission. Phys. Rev. B 2004, 69, 165317.

41 Schlawin, F.; Dorfman, K. E.; Fingerhut, B. P.; Mukamel, S. Manipulation of two-photoninduced fluorescence spectra of chromophore aggregates with entangled photons: A simulation study. Phys. Rev. A 2012, 86, 023851. 
42 Dayan, B. Theory of two-photon interactions with broadband down-converted light and entangled photons. Phys. Rev. A 2007, 76, 043813.

43 Schlawin, F.; Mukamel, S. Photon statistics of intense entangled photon pulses. J. Phys. B: At. Mol. Opt. 2013, 46, 175502.

44 Muthukrishnan, A.; Agarwal, G. S.; Scully, M. O. Inducing Disallowed Two-Atom Transitions with Temporally Entangled Photons. Phys. Rev. Lett. 2004, 93, 093002.

45 Richter, M.; Mukamel, S. Collective two-particle resonances induced by photon entanglement. Phys. Rev. A 2011, 83, 063805.

46 Schlawin, F.; Dorfman, K. E.; Fingerhut, B. P.; Mukamel, S. Suppression of population transport and control of exciton distributions by entangled photons. Nature Comm. 2013, 4, 1782 .

47 Oka, H. Selective two-photon excitation of a vibronic state by correlated photons. J. Chem. Phys. 2011, 134, 124313.

48 Oka, H. Control of vibronic excitation using quantum-correlated photons. J. Chem. Phys. 2011, 135, 164304 .

49 Schlawin, F. Quantum-Enhanced Nonlinear Spectroscopy; Springer Theses; Springer International Publishing, 2016.

50 Bernhard, C.; Bessire, B.; Feurer, T.; Stefanov, A. Shaping frequency-entangled qudits. Phys. Rev. A 2013, 88, 032322.

51 Bessire, B.; Bernhard, C.; Feurer, T.; Stefanov, A. Versatile shaper-assisted discretization of energy-time entangled photons. New J. Phys. 2014, 16, 033017.

52 Schlawin, F.; Buchleitner, A. Theory of coherent control with quantum light. New J. Phys. 2017, 19, 013009. 
53 Oka, H. Two-photon absorption by spectrally shaped entangled photons. Phys. Rev. A 2018, 97, 033814. 